\title{
Antidepressiva bei Kindern und Jugendlichen: Stellenwert von Johanniskraut-Extrakt
}

Die Analysen klinischer Studien mit klassischen Antidepressiva (Tri- und Tetrazyklika) bei Kindern und Jugendlichen ergaben einen ernüchternd geringen Nutzen, aber erhebliche Nebenwirkungen. Neuere Antidepressiva wie Selektive SerotoninWiederaufnahme-Hemmer schienen einen Ausweg zu bieten, bis man in klinischen Studien ein erhöhtes Risiko für Suizidversuche, feindseliges Verhalten und Selbstverletzungen entdeckte. In Metaanalysen erwiesen sich zudem die meisten Substanzen als nur marginal wirksam. Kein Antidepressivum wird heute weltweit als Goldstandard für die Therapie juveniler Depressionen angesehen. Bisher liegen zwar einige vielversprechende Anwendungsbeobachtungen und Pilotstudien mit Johanniskraut-Extrakten bei Kindern und Jugendlichen vor, kontrollierte klinische Studien fehlen jedoch noch und sind überfällig.

Die Verschreibungszahlen von psychotropen Arzneimitteln bei Kindern und Jugendlichen sind in den letzten Jahren weltweit kontinuierlich angestiegen, speziell Antidepressiva (AD) haben in den ersten Jahren des neuen Jahrtausends deutlich zugelegt. Die Einführung der Selektiven Serotonin-Wiederaufnahme-Hemmer (SSRI) anstelle der älteren Trizyklika (TCA) führte zu einer erheblichen Ausweitung der klinischen Anwendung von AD bei juvenilen Patienten. In Europa hat noch keines dieser neueren $\mathrm{AD}$ eine Zulassung für diesen Patientenkreis. Die relativ meisten Verordnungen erfolgen in den USA. Diese Entwicklung endete jedoch abrupt, nachdem die Europäische Zulassungsbehörde EMEA, die amerikanische FDA und andere nationale Behörden Warnungen herausgegeben hatten, SSRI und andere moderne $\mathrm{AD}$ könnten das Risiko für Suizidversuche und -vorstellungen, feindseliges Verhalten und Selbstverletzungen bei Kindern und Jugendlichen erhöhen. Die Warnungen waren verbunden mit der Empfehlung, diese Substanzen nur in dringenden Fällen einzusetzen und die Patienten während und nach der Behandlung sorgfältig auf die oben genannten Symptome hin zu beobachten.

\section{"Moderne" Antidepressiva}

bei Kindern und Jugendlichen - sicher und wirksam? Die nach 1990 eingeführten SSRI wurden von Kinder- und Jugendpsychiatern sehr begrüsst, da sie die unangenehmen Nebenwirkungen der klassischen AD (Müdigkeit, Mundtrockenheit, Gewichtszunahme u.a.) nicht oder in geringerem Ausmass aufwiesen. Der Umstieg auf die SSRI wurde noch begünstigt, weil Metaanalysen keine Überlegenheit von TCA gegenüber Placebo bei Depressionen im Kindes- und Jugendalter nachweisen konnten. Obwohl einige SSRI häufig bei juveniler Depression eingesetzt werden, ist in den USA bis heute nur Fluoxetin für diese Anwendung zugelassen. In den letzten Jahren ist dafür eine heftige Diskussion um Nutzen und Risiken von SSRI entbrannt.

Eine Metaanalyse von veröffentlichten und unveröffentlichten Studien erbrachte für die meisten SSRI ein ungünstiges Nutzen/Risiko-Verhältnis, mit Ausnahme von Fluoxetin und - eingeschränkt - Citalopram. Fluoxetin erwies sich in einer umfangreichen Untersuchung als wirksam. Noch besser war eine Kombination von Fluoxetin mit kognitiver Verhaltenstherapie, die allein weniger wirksam als Fluoxe-

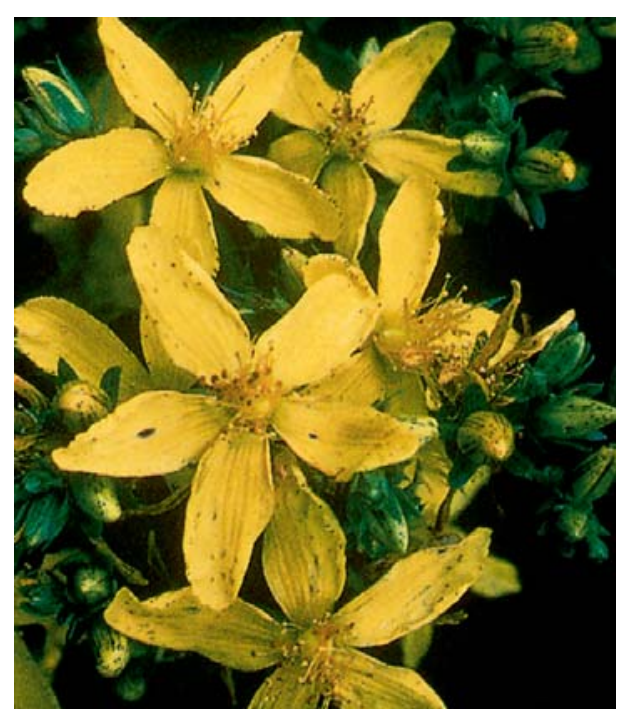

tin und nicht signifikant besser als Placebo war. Insbesondere war die Zahl der Ereignisse, die mit Suizid- und Selbstverletzungsgedanken verbunden waren, unter der Kombination niedriger als mit Fluoxetin allein. Neben diesen schwerwiegenden Nebenwirkungen wurden auch Entzugsphänomene wie Schwindel, Schlafstörungen und Angst nach plötzlichem Absetzen unter SSRI häufiger beobachtet als unter Placebo, während die Rate tatsächlich durchgeführter Suizide nicht erhöht war. Auswertungen klinischer Studien ergaben typischerweise eine Erhöhung dieses Risikos um ca. $70 \%$. Im Januar 2007 weitete die FDA ihre diesbezüglichen Warnungen auch auf junge Erwachsene aus.

Antidepressiva haben trotz dieser entmutigenden Bilanz unter klar definierten Bedingungen ihren Platz in der Depressionsbehandlung von Kindern und Jugendlichen, das gilt zumindest für Fluoxetin. Da aber auch auf diese Substanz ca. $40 \%$ der jugendlichen Patienten nicht ansprechen, besteht zweifelsfrei Bedarf an Alternativen, insbesondere bei leichten bis moderaten Fällen, in denen ein erhöhtes Suizidrisiko nicht hingenommen werden kann.

\section{Johanniskraut-Extrakt bei Depression von Kindern und Jugendlichen}

In älteren Lehrbüchern wird Johanniskraut besonders zur Behandlung von Bettnässen bei Kindern empfohlen und mit 
Tab. 1. Anwendungsbeobachtung mit Hypericum-Extrakt LI 160 bei Kindern unter 12 Jahren: Anzahl der Kinder mit spezifischen depressiven Symptomen zu Beginn der Untersuchung und Anteil vollständiger Remissionen nach Abschluss der Studie.

\begin{tabular}{|c|c|c|}
\hline Symptom & $\begin{array}{l}\text { Ausgangs- } \\
\text { wert (n) }\end{array}$ & $\begin{array}{l}\text { vollständige } \\
\text { Remission bei (\%) }\end{array}$ \\
\hline Konzentrationsstörungen & 90 & 51 \\
\hline Nervosität & 82 & 52 \\
\hline Schlafstörungen & 75 & 91 \\
\hline Reizbarkeit & 72 & 65 \\
\hline Freudlosigkeit & 63 & 86 \\
\hline depressive Stimmung & 61 & 87 \\
\hline Erschöpfung & 59 & 88 \\
\hline Antriebslosigkeit & 44 & 91 \\
\hline
\end{tabular}

seiner antidepressiven Wirkung begründet, die es als gute Alternative zu dem damals bei dieser Indikation gebräuchlichen Imipramin erscheinen liess. Entsprechend wurde Johanniskraut natürlich auch bei den später von der Kommission E definierten Beschwerden eingesetzt, nämlich depressiven Verstimmungszuständen, Angst/nervöser Unruhe und psychovegetativen Störungen; dass dies auch für den pädiatrischen Bereich galt, zeigten die entsprechenden Dosierungsempfehlungen für Kinder ab 4 Jahren.

\section{Anwendungsbeobachtung}

In einer multizentrischen Anwendungsbeobachtung an 101 Kindern unter 12 Jahren wurden Compliance, Verträglichkeit und Wirksamkeit des Johanniskrautextraktes LI 160 $\left(\operatorname{Jarsin}^{\circledR}\right)$ über vier Wochen geprüft, bei Bedarf war eine Verlängerung um 2 Wochen möglich. 15\% der Patienten erhielten $300 \mathrm{mg} / \mathrm{d}$ Extrakt, 26\% $600 \mathrm{mg} / \mathrm{d}$, 25\% nahmen die übliche Erwachsenendosierung von $900 \mathrm{mg} / \mathrm{d}$ und $3 \%$ sogar $1800 \mathrm{mg} / \mathrm{d}$. Studienabbrüche aufgrund von Nebenwirkungen gab es nicht. Ärzte und Eltern bewerteten die Verträglichkeit am Ende mit «gut» oder «sehr gut», 75\% der Patienten setzten die Einnahme bis zur 6. Woche fort.

Die Diagnosen wurden nicht entsprechend DSM oder ICD-Kriterien gestellt, sondern nach den von der Kommission E definierten Anwendungsgebieten. 47\% der Patienten litten unter depressiven Verstimmungen, ebenfalls $47 \%$ unter psychovegetativen Störungen, 48\% unter Ängsten und 54\% unter Unruhe. Nach vier Wochen beurteilten die Ärzte den Therapieerfolg bei $87 \%$ der Patienten mit «gut» oder «sehr gut», $3 \%$ waren unverändert, und in $10 \%$ wurden keine Angaben gemacht. Neben der globalen Beurteilung bewerteten die Ärzte auch die Veränderungen von acht typischen Depressionssymptomen. Am häufigsten waren Konzentrationsstörungen, Nervosität, Schlafstörungen und Reizbarkeit (Tab. 1). Bei Beendigung der Studie waren alle Symptome bei mindestens 95\% der Patienten gebessert oder ganz verschwunden. Reizbarkeit, Nervosität und Konzentrationsstörungen waren am schwierigsten nachhaltig zu beeinflussen.

\section{Pilotstudien}

Zwei offene Pilotstudien mit total 60 jugendlichen Patienten mit Major Depression nach DSM-IV benutzten eine speziell für juvenile Depression angepasste und validierte Skala, die Children's Depression Rating Scale - revised (CDRS-R). Diese Skala enthält unter anderem Bewertungen für schulische Leistungsstörungen, Unfähigkeit sich zu freuen, übermässiges Weinen, Hypoaktivität sowie morbide und suizidale Vorstellungen. Die Therapie mit Johanniskraut erwies sich an Hand der eindrucksvollen Score-Verbesserungen als vielversprechend, und fast alle Patienten wollten das Johanniskraut-Präparat nach Abschluss der Studien weiternehmen. Die Autoren schlossen aus ihren Ergebnissen, dass Johanniskrautpräparate für jugendliche Patienten mit «Major Depression» geeignet und effektiv seien und gut vertragen würden.

\section{Schlussfolgerungen}

Offensichtlich besteht ein wachsender Bedarf an einer wirksamen und sicheren Medikation für depressive Kinder und Jugendliche. Häufig ist die kognitive Verhaltenstherapie die Methode der Wahl, doch in vielen Fällen erhalten diese Patienten wegen zu geringer Kapazitäten und langer Wartelisten keinen Therapieplatz in angemessener Zeit. Selbst wenn dies der Fall ist, kann der Therapieeffekt durch Kombination mit einem Arzneimittel deutlich verbessert werden, wie Studien gezeigt haben. Die immer wieder geäusserten Zweifel an der Wirksamkeit und Sicherheit der neuen Antidepressiva dürften viele Ärzte davon abhalten, überhaupt eine medikamentöse Therapie anzuwenden, wenn sie nicht gleichzeitig eine intensive und kompetente psychiatrisch-psychotherapeutische Betreuung garantieren können.

In dieser Situation können Johanniskraut-Präparate einen Ausweg bieten. Ihre in zahlreichen klinischen Studien und Meta-Analysen an Erwachsenen demonstrierte gute Wirksamkeit und Verträglichkeit, die langjährigen traditionellen Erfahrungen sowie die Ergebnisse offener Studien an Kindern und Jugendlichen weisen auf ein vielversprechendes therapeutisches Potenzial hin. Kontrollierte klinische Studien mit diesen Patienten stehen aber noch aus. Sie sollten sich zunächst auf leichtere bis mittelschwere Fälle beschränken, bei denen kein suizidales Risiko besteht. Ärzte, die Johanniskraut verschreiben, können sich die hohe Akzeptanz pflanzlicher Arzneimittel seitens der Eltern zur Complianceförderung zunutze machen.

Bridge JA, lyengar S, Salary CB, Barbe RP, Birmaher B, Pincus HA, Ren L, Brent DA: Clinical response and risk for reported suicidal ideation and suicide attemps in pediatric antidepressant treatment: a meta-analysis of randomized controlled trials. JAMA 2007;297:168-1696.

Gibbons RD, Brown HC, Hur K, Marcus SM, Bhaumik DK, Erkens JA, Herings RMC, Mann JJ: Early evidence on the effects of regulators' suicidality warnings on SSRI prescriptions and suicide in children and adolescents. Am J Psychiatry 2007;164:1356-1363.

Seelinger G, Mannel M: Antidepressiva bei Kindern und Jugendlichen welchen Stellenwert hat Johanniskraut? Zeitschrift für Phytotherapie 2007; 28:162-168. 